\title{
Two-photon excited luminescence spectral distribution observation in wide-gap semiconductor crystals.
}

\begin{abstract}
The use of photoluminescence excited with two-photon process for characterizing the defect and impurity level in wide-gap semiconductor is discussed in this paper. Defects of polycrystalline zinc selenide ( $\mathrm{ZnSe}$ ) is observed deep inside the crystal. Two types of defects can be detected based on the spectral luminescence image. One type of defect can be seen in the entire spectrum images. Meanwhile, other types of defects can only be observed at higher energy of the spectrum, from 460 to $465 \mathrm{~nm}$. This study represents works of identifying crystals defect in wide gap materials by two-photon luminescence technique.
\end{abstract}

Keyword: Crystal defects; Photoluminescence; Photons; Polycrystalline materials; Semiconducting zinc compounds; Semiconductor materials. 